FEV $_{1}$ and SGRQ (Table 1). The benefit of SFC over SAL was seen with the composite endpoint in the ITT population and both GOLD subgroups.

Conclusion This post hoc analysis showed that, although most patients eventually experienced one of the three measures of deterioration, SFC significantly reduced the risk of a first composite CID compared to SAL. This added benefit of ICS was equally present in patients with mild/moderate or severe/very severe COPD.

\section{P123 INHALED CORTICOSTEROID PLUS LONG-ACTING $\beta 2$ - AGONIST THERAPY IS OVERUSED IN THE TREATMENT OF PATIENTS WITH CHRONIC OBSTRUCTIVE PULMONARY DISEASE: POST HOC ANALYSES OF TWO 1-YEAR STUDIES}

${ }^{1} \mathrm{H}$ Watz, ${ }^{2} \mathrm{GT}$ Ferguson, ${ }^{3} \mathrm{~L}$ Grönke, ${ }^{3} \mathrm{~F}$ VoB, ${ }^{4} \mathrm{R}$ Abrahams, ${ }^{5} \mathrm{R}$ Buhl. ${ }^{1}$ Pulmonary Research Institute at Lung Clinic Grosshansdorf, Airway Research Center North, Member of the German Center for Lung Research, Grosshansdorf, Germany; ${ }^{2}$ Pulmonary Research Institute of Southeast Michigan, Livonia, Michigan, USA; ${ }^{3}$ Boehringer Ingelheim Pharma GmbH \& Co. KG, Ingelheim, Germany; ${ }^{4}$ Morgantown Pulmonary Associates, Morgantown, West Virginia, USA; ${ }^{5}$ Pulmonary Department, Mainz University Hospital, Mainz, Germany

\subsection{6/thoraxjnl-2015-207770.260}

Rationale Inhaled corticosteroid (ICS) plus long-acting $\beta_{2}$-agonist (LABA) therapy is indicated for different patient groups with chronic obstructive pulmonary disease (COPD) in the USA and Europe. In the previous version of the Global initiative for chronic Obstructive Lung Disease (GOLD) recommendations, the use of ICS plus LABA therapy was restricted to patients with severe and very severe lung-function impairment and frequent exacerbations, with overtreatment in milder patient populations well documented. The current GOLD document recommends the use of ICS plus LABA maintenance therapy for patients in categories $\mathrm{C}$ and $\mathrm{D}$.

Methods We present post hoc analyses from the two pivotal 1year TONADO studies to assess the use of ICS plus LABA maintenance therapy in patients classified as GOLD A/B and C/D. As these studies were initiated before the update of the GOLD recommendations, no modified Medical Research Council Dyspnoea scale or COPD Assessment Test data were available to further classify these patients into categories A or B and C or D. Based on the reported COPD exacerbation history and lungfunction measurements, 2259 patients were classified as GOLD $\mathrm{A} / \mathrm{B}$ and 2903 as GOLD C/D. Baseline characteristics and concomitant medications at baseline are presented in Table 1 .

Results In the GOLD A/B subgroup, 7.3\% of patients were receiving treatment with ICS alone and $31.3 \%$ were receiving treatment with ICS plus LABA at study baseline. In the GOLD C/D subgroup, the incidences of patients receiving treatment with ICS alone and ICS plus LABA at study baseline were $8.8 \%$ and $45.5 \%$, respectively.

Conclusions Almost 40\% of patients classified as GOLD A/B are receiving treatment with ICS maintenance therapy, either alone, in free combination or as a fixed-dose combination therapy, despite GOLD recommendations for use only in patients with more severe lung-function impairment and frequent exacerbations. Our analyses confirm previous reports, highlighting that treatment regimens containing ICS therapy are being used early in the management of patients with COPD, which may not be appropriate based on current GOLD recommendations.

Funding Boehringer Ingelheim.

\begin{tabular}{lll} 
Abstract P123 Table 1 & & \\
\hline & GOLD A/B & GOLD CID \\
\hline $\mathrm{n}$ & 2259 & 2903 \\
Age (years), mean \pm SD & $64.3 \pm 8.6$ & $63.8 \pm 8.0$ \\
Male, $\mathrm{n}(\%)$ & $1576(69.8)$ & $2186(75.3)$ \\
Ex-smoker, $\mathrm{n}(\%)$ & $1348(59.7)$ & $1906(65.7)$ \\
Post-bronchodilator FEV ${ }_{1}^{(L), \text { mean } \pm \text { SD }}$ & $1.73 \pm 0.44$ & $1.1 \pm 0.37$ \\
Post-bronchodilator FEV ${ }_{1}(\%$ predicted), mean \pm SD & $62.9 \pm 8.2$ & $39.9 \pm 11.6$ \\
Reversibility (mL), mean \pm SD & $193 \pm 158$ & $154 \pm 131$ \\
Baseline maintenance medication, $\mathrm{n}(\%)$ & & \\
ICS & $871(38.6)$ & $1575(54.3)$ \\
ICS without LABA & $165(7.3)$ & $255(8.8)$ \\
ICS plus LABA & $706(31.3)$ & $1320(45.5)$ \\
LABA & $904(40.0)$ & $1489(51.3)$ \\
LAMA & $768(34.0)$ & $1072(36.9)$ \\
Xanthines & $153(6.8)$ & $363(12.5)$ \\
\hline
\end{tabular}

$\mathrm{SD}$, standard deviation; FEV ${ }_{1}$, forced expiratory volume in $1 \mathrm{~s}$; LAMA, long-acting muscarinic antagonist.

\section{P124 A RANDOMISED, PARALLEL-GROUP STUDY TO EVALUATE THE EFFECT OF UMECLIDINIUM ADDED TO INHALED CORTICOSTEROID/LONG-ACTING BETA- AGONIST COMBINATION THERAPY IN SUBJECTS WITH CHRONIC OBSTRUCTIVE PULMONARY DISEASE}

${ }^{1}$ AR Sousa, ${ }^{1} \mathrm{JH}$ Riley, ${ }^{2} \mathrm{~A}$ Church, ${ }^{1} \mathrm{CQ}$ Zhu, ${ }^{1} \mathrm{YS}$ Punekar, ${ }^{1}$ WA Fahy. ${ }^{1}$ GlaxoSmithKline, Stockley Park West, Middlesex, UK; ${ }^{2}$ GlaxoSmithKline, 5 Moore Drive, Research Triangle Park, NC, USA

\subsection{6/thoraxjnl-2015-207770.261}

Rationale To evaluate efficacy and safety of adding umeclidinium (UMEC), a long-acting muscarinic antagonist (LAMA), to inhaled corticosteroid (ICS)/long-acting $\beta$-agonist (LABA) in patients with moderate-to-very severe chronic obstructive pulmonary disease (COPD) for 12-weeks.

Methods Multicentre, randomised, double-blind, parallel-group study. Inclusion criteria included diagnosis of COPD, modified Medical Research Council Dyspnoea Scale score $\geq 2$ (i.e. patients symptomatic on ICS/LABA), post-salbutamol forced expiratory volume in one second $\left(\mathrm{FEV}_{1}\right) \leq 70 \%$ predicted and $\mathrm{FEV}_{1} /$ forced vital capacity ratio of 1 at Day 85; other endpoints included 0-6 h weighted mean $\mathrm{FEV}_{1}$, rescue medication use, COPD assessment test (CAT) score, and transition dyspnoea index (TDI) score. Adverse events (AEs) were also investigated.

Results In the UMEC+ICS/LABA and $\mathrm{PBO}+\mathrm{ICS} / \mathrm{LABA}$ groups, 119 and 117 patients were randomised, respectively, receiving fluticasone/salmeterol (40\%), budesonide/formoterol (43\%), and other ICS/LABA, including generics (17\%). Compared with PBO + ICS/LABA, UMEC+ICS/LABA resulted in statistically significant improvements in change from baseline trough $\mathrm{FEV}_{1}$ at Day 85 and $0-6$ h weighted mean $\mathrm{FEV}_{1}$ at Day 84 (Table 1). UMEC $+\mathrm{ICS} / \mathrm{LABA}$ resulted in a statistically significant reduction in change from baseline mean puffs/day of rescue salbutamol over Weeks 1-12 versus $\mathrm{PBO}+\mathrm{ICS} / \mathrm{LABA}$, but not for percentage of rescue-free days. Change from baseline in CAT score at Day 84 was statistically significantly different for UMEC+ICS/LABA versus $\mathrm{PBO}+\mathrm{ICS} / \mathrm{LABA}$, but TDI score was not significantly different for UMEC+ICS/LABA versus $\mathrm{PBO}+\mathrm{ICS} / \mathrm{LABA}$; the study was not powered for these endpoints. Incidence of AEs was similar with UMEC+ICS/LABA and PBO+ICS/LABA; $\mathrm{n}=45(38 \%)$ and $n=49(42 \%)$, respectively. The most common AEs were nasopharyngitis (13-15\%) and headache (3-7\%). 
Abstract P124 Table 1 Endpoint results

\begin{tabular}{|c|c|c|c|}
\hline & $\begin{array}{l}\text { UMEC + ICS/LABA } \\
\mathrm{N}=119)\end{array}$ & $\begin{array}{l}\mathrm{PBO}+\mathrm{ICS} / \mathrm{LABA} \\
(\mathrm{N}=117)\end{array}$ & $\begin{array}{l}\text { Treatment } \\
\text { Diff. vs PBO } \\
(95 \% \mathrm{Cl})\end{array}$ \\
\hline Trough FEV1 at Day 85 & $\mathrm{n}=109$ & $\mathrm{n}=110$ & \\
\hline $\begin{array}{l}\text { LS mean change from } \\
\text { baseline, L (SE) }\end{array}$ & $0.090(0.0183)$ & $-0.033(0.0184)$ & $\begin{array}{l}0.123^{\mathrm{a}} \\
(0.071,0.174)\end{array}$ \\
\hline $\begin{array}{l}0-6 \text { hours weighted } \\
\text { mean FEV1 at Day } 84\end{array}$ & $\mathrm{n}=107$ & $\mathrm{n}=110$ & \\
\hline $\begin{array}{l}\text { LS mean change from } \\
\text { baseline, L (SE) }\end{array}$ & $0.184(0.0176)$ & $0.035(0.0175)$ & $\begin{array}{l}0.148^{\mathrm{a}} \\
(0.099,0.197)\end{array}$ \\
\hline $\begin{array}{l}\text { Rescue use (mean } \\
\text { puffs/day)* }\end{array}$ & $\mathrm{n}=119$ & $\mathrm{n}=116$ & \\
\hline $\begin{array}{l}\text { LS mean change from } \\
\text { baseline (SE) }\end{array}$ & $-0.53(0.105)$ & $-0.15(0.106)$ & $\begin{array}{l}-0.38^{b} \\
(-0.67,-0.10)\end{array}$ \\
\hline $\begin{array}{l}\text { Rescue use }(\% \\
\text { rescue-free days) }{ }^{*, * *}\end{array}$ & $\mathrm{n}=119$ & $\mathrm{n}=116$ & \\
\hline Median (range) & $76.8(0,100)$ & $62.9(0,100)$ & $0^{c}(0.0,3.4)$ \\
\hline CAT score at Day 84 & $\mathrm{n}=110$ & $\mathrm{n}=110$ & \\
\hline $\begin{array}{l}\text { LS mean change from } \\
\text { baseline (SE) }\end{array}$ & $-0.37(0.457)$ & $0.94(0.457)$ & $\begin{array}{l}-1.31^{b} \\
(-2.59,-0.04)\end{array}$ \\
\hline TDI score at Day 84 & $\mathrm{n}=105$ & $\mathrm{n}=109$ & \\
\hline LS mean (SE) & $1.07(0.197)$ & $0.67(0.195)$ & $\begin{array}{l}0.40 \\
(-0.15,0.95)\end{array}$ \\
\hline
\end{tabular}

${ }^{a} p<0.001 ;{ }^{b} p<0.05 ;{ }^{c} p=0.640$, non-parametric analysis; *use over 1-12 weeks; **nonparametric analysis of $\%$ rescue-free days was a post hoc analysis and replaced the pre-specified analysis (percentage rescue-free day datadid not satisfy normality assumptions for MMRM analysis); improvements in CAT scores are shown by negative changes.
Conclusions UMEC+ICS/LABA improved lung function and reduced rescue medication use (mean puffs/day) and CAT score in patients with COPD versus $\mathrm{PBO}+\mathrm{ICS} / \mathrm{LABA}$. No additional safety concerns were identified with UMEC+ICS/LABA.

Funded by GlaxoSmithKline (NCT02257372).

COI Statement ARS, JR, AC, WAF, CQZ and YSP are employees of GSK and hold stocks/shares in GSK.

\section{P125 TIOTROPIUM PLUS OLODATEROL COMBINATION THERAPY PROVIDES LUNG-FUNCTION BENEFITS WHEN COMPARED TO TIOTROPIUM ALONE, IRRESPECTIVE OF PRIOR TREATMENT WITH A LONG-ACTING BRONCHODILATOR: POST HOC ANALYSES OF TWO 1-YEAR STUDIES}

${ }^{1} \mathrm{R}$ Buhl, ${ }^{2} \mathrm{R}$ Abrahams, ${ }^{3} \mathrm{~L}$ Grönke, ${ }^{3} \mathrm{~L}$ Korducki, ${ }^{4} \mathrm{M}$ Fležar, ${ }^{5} \mathrm{GT}$ Ferguson. ${ }^{1}$ Pulmonary Department, Mainz University Hospital, Mainz, Germany; ${ }^{2}$ Morgantown Pulmonary Associates, Morgantown, West Virginia, USA; ${ }^{3}$ Boehringer Ingelheim Pharma GmbH \& Co. $K G$, Ingelheim, Germany; ${ }^{4}$ Klinika Golnik, Golnik, Slovenia; ${ }^{5}$ Pulmonary Research Institute of Southeast Michigan, Livonia, Michigan, USA

\subsection{6/thoraxjnl-2015-207770.262}

Rationale Tiotropium plus olodaterol $(\mathrm{T}+\mathrm{O})$ is a novel oncedaily combination of the long-acting muscarinic antagonist (LAMA) tiotropium (T) and the recently approved long-acting $\beta_{2}$-agonist (LABA) olodaterol, for use as maintenance treatment in chronic obstructive pulmonary disease (COPD). These post hoc analyses of data from the two pivotal 1-year TONADO studies determined whether treatment with a long-acting

\section{Abstract P125 Table 1}

\begin{tabular}{|c|c|c|c|c|c|c|}
\hline & \multicolumn{3}{|c|}{ No prior LABD treatment } & \multicolumn{2}{|c|}{ Prior LABD treatment } & \\
\hline & \multicolumn{6}{|c|}{ All } \\
\hline & $\mathrm{T}+0$ & 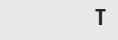 & & & & \\
\hline \multicolumn{7}{|l|}{ Baseline characteristics } \\
\hline $\mathrm{n}$ & 426 & & 454 & 603 & & 579 \\
\hline Age (years), mean $\pm S D$ & $62.7 \pm 8.3$ & & $62.6 \pm 8.8$ & $64.6 \pm 8.3$ & & $64.8 \pm 8.2$ \\
\hline Male, n (\%) & $291(68.3)$ & & $338(74.4)$ & $442(73.3)$ & & $417(72.0)$ \\
\hline Ex-smoker, n (\%) & $241(56.6)$ & & $278(61.2)$ & $388(64.3)$ & & $385(66.5)$ \\
\hline Post-bronchodilator $\mathrm{FEV}_{1}(\mathrm{~L})$, mean $\pm \mathrm{SD}$ & $1.38 \pm 0.52$ & & $1.43 \pm 0.54$ & $1.32(0.50)$ & & $1.32(0.51)$ \\
\hline Post-bronchodilator $\mathrm{FEV}_{1}(\%$ predicted), mean $\pm \mathrm{SD}$ & $50.7 \pm 16.0$ & & $50.8 \pm 15.7$ & $48.3 \pm 14.7$ & & $48.8 \pm 15.6$ \\
\hline Reversibility $(\mathrm{mL})$, mean $\pm \mathrm{SD}$ & $158 \pm 160$ & & $166 \pm 144$ & $169 \pm 138$ & & $174 \pm 147$ \\
\hline \multicolumn{7}{|l|}{ Lung function after 24 weeks of treatment } \\
\hline Adjusted mean $\mathrm{FEV}_{1} \mathrm{AUC}_{0-3,}(\mathrm{~mL}) \pm \mathrm{SE}$ & $270 \pm 1$ & & $154 \pm 9$ & $256 \pm 8$ & & $150 \pm 8$ \\
\hline \multirow[t]{3}{*}{ Adjusted mean trough $\mathrm{FEV}_{1}(\mathrm{~mL}) \pm \mathrm{SE}$} & $148 \pm 1$ & & $72 \pm 1$ & $134 \pm 8$ & & $86 \pm 8$ \\
\hline & \multicolumn{6}{|c|}{ GOLD 2} \\
\hline & $\mathrm{T}+0$ & $\mathbf{T}$ & $\mathrm{T}+0$ & & $\mathbf{T}$ & \\
\hline \multicolumn{7}{|l|}{ Baseline characteristics } \\
\hline $\mathrm{n}$ & 228 & 240 & 274 & & 277 & \\
\hline Age (years), mean $\pm S D$ & $63.0 \pm 8.2$ & $62.5 \pm 8.9$ & $65.3 \pm 8.9$ & & $64.8 \pm 8.7$ & \\
\hline Male, n (\%) & $148(64.9)$ & $160(66.7)$ & $202(73.7)$ & & $180(65.0)$ & \\
\hline Ex-smoker, n (\%) & $117(51.3)$ & $128(53.3)$ & $176(64.2)$ & & $179(64.6)$ & \\
\hline Post-bronchodilator $\mathrm{FEV}_{1}(\mathrm{~L})$, mean $\pm \mathrm{SD}$ & $1.72 \pm 0.44$ & $1.77(0.47)$ & $1.69(0.44)$ & & $1.69(0.45)$ & \\
\hline Post-bronchodilator $\mathrm{FEV}_{1}(\%$ predicted), mean $\pm \mathrm{SD}$ & $63.2 \pm 8.8$ & $63.1 \pm 8.4$ & $62.0 \pm 7.4$ & & $62.7 \pm 8.1$ & \\
\hline Reversibility $(\mathrm{mL})$, mean \pm SD & $175 \pm 156$ & $184 \pm 163$ & $201 \pm 151$ & & $196 \pm 170$ & \\
\hline \multicolumn{7}{|l|}{ Lung function after 24 weeks of treatment } \\
\hline Adjusted mean $\mathrm{FEV}_{1} \mathrm{AUC}_{0-3,}(\mathrm{~mL}) \pm \mathrm{SE}$ & $289 \pm 13$ & $175 \pm 13$ & $302 \pm(12)$ & & $179 \pm 12$ & \\
\hline Adjusted mean trough $\mathrm{FEV}_{1}(\mathrm{~mL}) \pm \mathrm{SE}$ & $146 \pm 14$ & $68 \pm 14$ & $156 \pm 13$ & & $95 \pm 13$ & \\
\hline
\end{tabular}

$\mathrm{SD}$, standard deviation; SE, standard error. 